\title{
SIROLIMUS (RAPAMYCIN) POTENTIATES CYCLOSPORINE IN PREVENTION OF ACUTE LUNG REJECTION
}

James Longoria, MD

Randall F. Roberts, MD

Charles C. Marboe, MD

Bruce C. Stouch, MS

Vaughn A. Starnes, MD

Mark L. Barr, MD
Background: Cyclosporine-based immunosuppressive regimens (INN: ciclosporin) in human lung transplantation continue to result in a high incidence of acute cellular rejection. We investigated the use of sirolimus, a macrolide with structural similarity to tacrolimus, as monotherapy and in combination with cyclosporine in a rodent lung transplant model. Methods: Orthotopic left lung transplantation was performed in Lewis recipients from Brown-Norway donor rats with syngeneic Lewis-to-Lewis controls. Open biopsies were performed on postoperative day 7, and the severity of acute lung rejection was graded by a pathologist blinded to the protocol. Results: All recipients survived despite the amount of acute rejection seen on examination of the biopsy tissue. Lewis-to-Lewis isografts demonstrated near normal pulmonary architecture. Allogeneic recipients receiving high-dose cyclosporine (25 $\mathrm{mg} / \mathrm{kg}$ ) monotherapy showed mild to moderate acute rejection with some perivascular focal interstitial infiltrates. Recipients receiving lowdose cyclosporine $(5 \mathrm{mg} / \mathrm{kg}$ ) monotherapy or low- or high-dose sirolimus ( 0.5 or $2.0 \mathrm{mg} / \mathrm{kg}$, respectively) monotherapy demonstrated massive cellular infiltration leading to necrosis and infarction and could not be graded. However, the addition of low-dose sirolimus $(0.5 \mathrm{mg} / \mathrm{kg})$ to lowdose cyclosporine $(5 \mathrm{mg} / \mathrm{kg})$ demonstrated a significant potentiating immunosuppressive effect, and the addition of high-dose sirolimus $(2.0$ $\mathrm{mg} / \mathrm{kg})$ to low-dose cyclosporine $(5.0 \mathrm{mg} / \mathrm{kg})$ demonstrated an even greater effect, with rejection scores better than those obtained with high-dose cyclosporine monotherapy and similar to those obtained with isografts. Conclusions: This study demonstrates that low-dose sirolimus has a cyclosporine-sparing effect and that a higher dose of sirolimus in combination with cyclosporine strongly protects lung allografts from acute cellular rejection. These results suggest that sirolimus may be indicated as an adjunct to current cyclosporine-based immunosuppressive regimens in clinical lung transplantation. (J Thorac Cardiovasc Surg 1999;117:714-8) ung transplantation has become an accepted form of treatment for many types of end-stage lung disease. This has been a consequence, in part, of improvements in organ preservation, surgical technique, postoperative care, and immunosuppression. Despite these improvements, lung transplantation continues to be plagued by

From the Division of Cardiothoracic Surgery, University of Southern California and Childrens Hospital Los Angeles, Los Angeles, Calif.

Supported by a grant to Dr Barr from the Heart and Lung Surgery Foundation, Los Angeles, Calif.

Received for publication Aug 7, 1998; revisions requested Oct 30, 1998; revisions received Nov 10, 1998; accepted for publication Nov 11, 1998. a higher incidence of rejection and infection than other solid organ transplants. Most current clinical protocols include some combination of cyclosporine (INN: ciclosporin) (or most recently tacrolimus), corticosteroids, azathioprine, and occasionally antilymphocyte preparations. Although the use of cyclosporine has allowed

\footnotetext{
Address for reprints: Mark L. Barr, MD, Division of Cardiothoracic Surgery, University of Southern California, 1510 San Pablo St, Los Angeles, CA 90033.

Copyright (C) 1999 by Mosby, Inc.
}

$0022-5223 / 99 \$ 8.00+0 \quad \mathbf{1 2 / 1 / 9 5 9 2 6}$ 
tremendous advances in transplantation, acute allograft rejection and subsequently the long-term complication of obliterative bronchiolitis remain major obstacles in clinical lung transplantation. New immunosuppressive agents or combinations of agents for pulmonary allografts need to be evaluated.

Sirolimus is a macrolide isolated from the actinomycete Streptomyces hygroscopicus with some structural similarity to tacrolimus. ${ }^{1}$ The drug acts between the $\mathrm{G} 1$ and $\mathrm{S}$ phase of the T-cell activation cascade, can act directly on both T cells and B cells, and can strongly suppress in vitro immunoglobulin production., ${ }^{2,3} \mathrm{Al}-$ though structurally related to tacrolimus, sirolimus does not inhibit interleukin-2 production but inhibits the proliferative response of $\mathrm{T}$ cells to interleukin- 2 and other cytokines. Sirolimus has been used as an effective immunosuppressive agent after skin, heterotopic and vascularized heart, pancreas, kidney, and small bowel transplantation in the rat. ${ }^{1,4}$ However, sirolimus has not been used in clinical lung transplantation, in part, because of the paucity of its use in animal transplant models.

We investigated the addition of sirolimus to cyclosporine and hypothesized that this immunosuppressive combination would result in an additive immunosuppressive effect and demonstrate a cyclosporine-sparing effect in a rat model of orthotopic acute lung transplant rejection.

\section{Materials and methods}

Orthotopic left lung transplantation was performed in Lewis recipients from Brown-Norway donor rats (Charles River, NJ). Animals were assigned into 8 groups. Syngeneic transplantation of Lewis to Lewis without any immunosuppression (group $\mathrm{A}, \mathrm{n}=8$ ) served as a "negative" control. Allogeneic transplantation of Brown-Norway to Lewis without any immunosuppression (group $\mathrm{B}, \mathrm{n}=6$ ) served as a "positive" control. There were 6 experimental groups (total $\mathrm{n}$ =37) randomly assigned to receive immunosuppressive therapy with intramuscular cyclosporine (Cremaphor-based, Sandimmune, Novartis Pharma, East Hanover, NJ), monotherapy (group C, $5 \mathrm{mg} / \mathrm{kg}$ per day; group D, $25 \mathrm{mg} / \mathrm{kg}$ per day); intraperitoneal sirolimus (Wyeth-Ayerst Research Laboratories, Philadelphia, Pa) monotherapy (group E, 0.5 $\mathrm{mg} / \mathrm{kg}$ per day; group F, $2.0 \mathrm{mg} / \mathrm{kg}$ per day); or a combination of cyclosporine plus sirolimus (group G, $5 \mathrm{mg} / \mathrm{kg}$ per day $/ 0.5$ $\mathrm{mg} / \mathrm{kg}$ per day; group $\mathrm{H}, 5 \mathrm{mg} / \mathrm{kg}$ per day $/ 2.0 \mathrm{mg} / \mathrm{kg}$ per day). All animals received humane care in compliance with the "Principles of Laboratory Animal Care" published by the National Society for Medical Research and the "Guide for the Care and Use of Laboratory Animals" (National Institutes of Health publication No. 86-23, revised 1985).

All animals $(\mathrm{n}=51)$ underwent left orthotopic lung transplantation by means of the microvascular technique described previously in the literature..$^{5,6}$ The donor procedure was performed concurrently to minimize the ischemic period of the pulmonary allograft. Donor rats were lightly anesthetized by inhalation of methoxyflurane in a sealed glass enclosure. The rats were premedicated by intramuscular injection of atropine $(0.25 \mathrm{mg} / \mathrm{kg})$, and operative anesthesia was achieved with intraperitoneal administration of pentobarbital $(50 \mathrm{mg} / \mathrm{kg})$. A tracheostomy was performed and a 14-gauge angiocatheter was inserted to institute positive-pressure ventilation. The animal was connected to a rodent ventilator that was adjusted to maintain normal ventilation (respiratory rate 70 breaths/ min; tidal volume $10 \mathrm{ml} / \mathrm{kg}$ body weight; peak inspiratory pressure $20 \mathrm{~cm} \mathrm{H}_{2} \mathrm{O}$ ). Through a median sternotomy, the thoracic organs were exposed and the inferior pulmonary ligaments incised. The donor animals received 300 units of intravenous heparin. A 16-gauge catheter was inserted into the main pulmonary artery through the right ventricular outflow tract. The inferior and superior venae cavae were divided and the left atrial appendage amputated. The pulmonary artery was then flushed with cold Euro-Collins solution $(100 \mathrm{ml} / \mathrm{kg})$ from a height of $30 \mathrm{~cm}$, and the thorax was irrigated with cold $\left(4^{\circ} \mathrm{C}\right)$ normal saline solution. A hemostatic clip was placed across the trachea to maintain lung inflation and the donor heart and lungs were removed en bloc. The left lung was dissected by dividing the hilar vessels and left main bronchus as proximally as possible to obtain sufficient length for the anastomosis. The left lung was kept inflated by placing a hemostatic clip across the proximal left main bronchus. The left lung block was then wrapped in a bed of gauze soaked with preservation solution and placed in a plastic container surrounded by ice and maintained at $4^{\circ} \mathrm{C}$ for the remainder of the ischemic storage period.

Recipient rats were lightly anesthetized by inhalation of methoxyflurane in a sealed glass enclosure. The rats were premedicated by intramuscular injection of atropine $(0.25$ $\mathrm{mg} / \mathrm{kg}$ ), and operative anesthesia was achieved with intraperitoneal administration of pentobarbital $(50 \mathrm{mg} / \mathrm{kg})$. The animal was orally intubated with a 14-gauge angiocatheter, placed in the right lateral decubitus position, and then connected to a rodent ventilator (respiratory rate 70 breaths/min; tidal volume $10 \mathrm{ml} / \mathrm{kg}$ body weight; peak inspiratory pressure $20 \mathrm{~cm} \mathrm{H}_{2} \mathrm{O}$ ) with operative anesthesia maintained with halothane $0.5 \%$ to $1.0 \%$. A left thoracotomy was performed at the fifth intercostal space. The inferior pulmonary ligament was incised and the hilar structures were dissected. The left pulmonary artery, left pulmonary vein, and left main stem bronchus were clamped with microvascular clamps and the native left lung was excised. The pulmonary vein and artery were anastomosed at the hilum sequentially with $10-0$ and 90 nylon sutures, respectively, in an interrupted fashion. The vascular clamps were then removed and reperfusion was established. The bronchial anastomosis was completed with a 9-0 nylon continuous suture. The lungs were then hyperinflated to remove atelectasis from the donor lung. A tube thoracostomy was then placed with the use of a 14-gauge silicone rubber catheter, and the thoracotomy incision was 
Table I. Effects of low- and high-dose cyclosporine and sirolimus monotherapy and combination therapy on lung allograft rejection scores

\begin{tabular}{lcclccc}
\hline & & & & & \multicolumn{2}{c}{ Rejection grade } \\
\cline { 3 - 6 } Group & Donor & Recipient & \multicolumn{1}{c}{ Immunosuppression } & Rejection grade (mean \pm SD) & Median & Range \\
\hline A & LEW & LEW & None & $0.25 \pm 0.46$ & 0 & $0-1$ \\
B & BN & LEW & None & X & X & X \\
C & BN & LEW & CsA $5 \mathrm{mg} / \mathrm{kg}$ & $2.71 \pm 0.49$ & 3 & $2-3$ \\
D & BN & LEW & CsA $25 \mathrm{mg} / \mathrm{kg}$ & $\mathrm{X}$ & $\mathrm{X}$ \\
E & BN & LEW & Siro $0.5 \mathrm{mg} / \mathrm{kg}$ & $\mathrm{X}$ & $\mathrm{X}$ \\
F & BN & LEW & Siro $2.0 \mathrm{mg} / \mathrm{kg}$ & $2.29 \pm 0.76$ & 2 \\
G & BN & LEW & CsA $5 \mathrm{mg} / \mathrm{kg}+$ Siro $0.5 \mathrm{mg} / \mathrm{kg}$ & $0.25 \pm 0.50$ & $1-3$ \\
H & BN & LEW & CsA $5 \mathrm{mg} / \mathrm{kg}+$ Siro $2.0 \mathrm{mg} / \mathrm{kg}$ & 0 & $0-1$ \\
\hline
\end{tabular}

$B N$, Brown-Norway; $L E W$, Lewis: $C s A$, cyclosporine; Siro, sirolimus; $X$, complete rejection of graft with massive cellular infiltration and could not be assigned a grade. Group A versus group D $(P<.001)$; group A versus group $\mathrm{G}(P=.002)$; group A versus group $\mathrm{H}(P=1.0)$. Group D versus group $\mathrm{G}(P=.592)$; group D versus group $\mathrm{H}(P=.003)$. Group $\mathrm{G}$ versus group $\mathrm{H}(P=.018)$.

closed in 3 layers. When the rats resumed spontaneous respiration, the tube thoracostomy and orotracheal tube were removed. The animals were allowed to recover after the operation in a heated, humidified chamber. The animals were then placed in clean cages after their recovery period and were provided food and water ad libitum.

The recipients received immunosuppression as previously outlined, and open lung biopsies were then performed on postoperative day 7 . The open lung biopsy tissue was snap frozen in liquid nitrogen, and frozen section analysis with hematoxylin and eosin staining was performed. Acute lung rejection was graded by a pathologist blinded to the protocol, who was using a modified version of the Working Formulation Lung Rejection Study Group ${ }^{7}$ : 0, no significant abnormality; 1, minimal acute rejection; 2 , mild acute rejection; 3 , moderate acute rejection; 4, severe acute rejection; and X, complete rejection of the graft with massive cellular infiltration that could not be assigned a grade. Rejection scores were then tabulated and analyzed.

Groups were compared by means of Fisher's exact test (2tailed) based on a hypergeometric distribution. Pairwise comparisons of the various treated groups versus the syngeneic control group were considered significant if the $P$ value was less than .0167 (traditional $\alpha=.05 / 3$ pairwise comparisons). Pairwise comparisons between the treated groups were considered significant if the $P$ value was less than .05 .

\section{Results}

All recipients survived despite the amount of acute rejection seen on biopsy tissue. Biopsy rejection scores are tabulated and represented in Table I. Syngeneic Lewis-to-Lewis transplants had a mean rejection score ( \pm standard deviation) of $0.25 \pm 0.46($ median $=0$ ) and demonstrated near normal pulmonary architecture. Brown-Norway-to-Lewis allografts without immunosuppression had massive cellular infiltration leading to infarction and necrosis within 7 days. Recipients receiving cyclosporine $25 \mathrm{mg} / \mathrm{kg}$ monotherapy had a rejection score of $2.71 \pm 0.49$ (median $=3$ ), demonstrating a mild to moderate amount of acute rejection with some perivascular focal interstitial infiltrates. Recipients receiving monotherapy consisting of cyclosporine $5 \mathrm{mg} / \mathrm{kg}$, sirolimus $0.5 \mathrm{mg}$, or sirolimus $2.0 \mathrm{mg}$ demonstrated massive cellular infiltration leading to necrosis and infarction and could not be graded. The addition of low-dose sirolimus $0.5 \mathrm{mg} / \mathrm{kg}$ to low-dose cyclosporine $5 \mathrm{mg} / \mathrm{kg}$ demonstrated a significant potentiating immunosuppressive effect with a mean rejection score of $2.29 \pm 0.76$ (median $=2$ ), which was not statistically different from that of the high-dose cyclosporine monotherapy group $(P=.592)$. Furthermore, the addition of high-dose sirolimus at $2.0 \mathrm{mg} / \mathrm{kg}$ to low-dose cyclosporine at $5.0 \mathrm{mg} / \mathrm{kg}$ demonstrated an even greater protective effect with a mean rejection score of $0.25 \pm 0.50$ (median $=0$ ), which is superior to low-dose sirolimus/low-dose cyclosporine therapy $(P=$ $.018)$, superior to high-dose cyclosporine monotherapy $(P=.003)$, and similar to results in isograft controls $(P=1.0)$.

\section{Discussion}

With improvements in survival, lung transplantation has become an accepted therapeutic option for patients with end-stage pulmonary disease. Cyclosporine-based immunosuppression protocols have resulted in a greater than $70 \%$ 1-year survival at major lung transplant centers. ${ }^{8}$ Despite these impressive results, the incidence of acute rejection episodes during the first 100 postoperative days is greater than $75 \%$ incidence. Infection continues to be the leading cause of death during the first year and appears to be due in part to the treatment of these acute rejection episodes. As has been 
previously demonstrated, ${ }^{9}$ obliterative bronchiolitis is the leading cause of death in patients surviving more than 1 year after transplantation, and acute rejection is one of the most significant risk factors and may be a marker for the development of obliterative bronchiolitis. ${ }^{9,10}$ It is apparent that what is required for continued improvement in long-term survival of recipients of lung transplants are immunosuppressive drug combinations that maximize immunosuppressive efficacy and minimize toxicity.

The present study examined the addition of sirolimus to cyclosporine in an orthotopic lung transplant model. To our knowledge, this is the first study in which this immunosuppressive combination has been used in a fully vascularized rat orthotopic lung transplant model. The results demonstrate that effective immunosuppression can be obtained when sirolimus is used in combination with cyclosporine and that the lung allograft is strongly protected from acute cellular rejection, as evidenced by histologic evaluation. Ochiai and associates ${ }^{11}$ demonstrated in a rat cardiac allograft model that sirolimus at $0.1 \mathrm{mg} / \mathrm{kg}$ for 11 days had a marginal effect on mean survival time, whereas the addition of cyclosporine at $3.2 \mathrm{mg} / \mathrm{kg}$ to sirolimus resulted in indefinite graft survival time. Similarly, Chen and coworkers ${ }^{12}$ demonstrated that the combination of cyclosporine 2 $\mathrm{mg} / \mathrm{kg}$ and sirolimus $0.08 \mathrm{mg} / \mathrm{kg}$ significantly prolonged the survival time of pancreaticoduodenal allografts in the rat when compared with low-dose therapy of these agents individually. Our data demonstrate the effectiveness of sirolimus administered at $2 \mathrm{mg} / \mathrm{kg}$ when used in combination with cyclosporine $5 \mathrm{mg} / \mathrm{kg}$, with rejection scores that are similar to those of lung isografts.

The toxicity of cyclosporine, in particular impaired renal function and hypertension, limits increases in dosages to prevent rejection. Therefore any immunosuppressive regimen that can maximize the net therapeutic index of cyclosporine while limiting its toxicity by having a sparing effect would be beneficial. The ability of small doses of sirolimus to produce synergistic immunosuppression has been evaluated in human lymphocytes in vitro. ${ }^{13}$ Knight and colleagues ${ }^{14}$ demonstrated consistent prolongation of renal allograft survival in the mongrel canine model. Sirolimus at 0.05 $\mathrm{mg} / \mathrm{kg}$ per day was added to subtherapeutic doses of cyclosporine, and this was found to potentiate the immunosuppressive effect in this model, with a mean allograft survival time of $75 \pm 14.3$ days. This synergistic effect was again demonstrated in a study of heart and kidney allograft models in rats. ${ }^{15}$ In every sirolimus/cyclosporine dose combination, allografts survived significantly longer than in recipients treated with a single drug. Furthermore, increased sirolimus/ cyclosporine doses resulted in increased prolongation of heart allograft survival. Similar results were obtained in our present study, in which the addition of low-dose sirolimus $(0.5 \mathrm{mg} / \mathrm{kg})$ to low-dose cyclosporine $(5 \mathrm{mg} / \mathrm{kg})$ resulted in lung allograft rejection scores similar to those of high-dose cyclosporine. In addition, the potentiating and cyclosporine-sparing effects were demonstrated when the sirolimus dose was increased to $2.0 \mathrm{mg} / \mathrm{kg}$, with rejection scores significantly better than those obtained with high-dose cyclosporine and similar to those obtained with isografts.

As demonstrated previously, ${ }^{4}$ sirolimus at intravenous doses as low as $0.08 \mathrm{mg} / \mathrm{kg}$ per day significantly prolonged cardiac and renal allograft survival, whereas $0.8 \mathrm{mg} / \mathrm{kg}$ per day was necessary to prolong small bowel allograft survival in rats. Sirolimus therapy showed a dose-dependent response with the highest tested dose of $0.8 \mathrm{mg} / \mathrm{kg}$ producing heart allograft survival of $48 \pm 3.6$ days. This prior work demonstrated the efficacy of sirolimus as monotherapy in the rat heart, kidney, pancreas, and small bowel transplant models. However, in our study, a similar efficacy could not be demonstrated in the rat orthotopic lung transplant model. Low and high doses of sirolimus were used as monotherapy and resulted in complete rejection of the lung allograft in each study group. Perhaps the lung is a more immunogenic organ allograft and requires more intensive immunosuppressive regimens. This might explain the higher incidence of rejection in clinical lung transplants when compared with other solid organ transplants.

Limitations of this study include the fact that this was purely a study of acute rejection. We investigated only one aspect of acute rejection, that being histologic evaluation. It remains to be investigated whether the additive effect of sirolimus to cyclosporine correlates with long-term graft acceptance. Another limitation of the study was that it did not evaluate the function of the allograft. Although the graft appeared to be viable on sectioning, with the appearance of normal pulmonary architecture, it was not determined whether indeed the graft retained its gas exchange capabilities. Finally, combining sirolimus with tacrolimus was not evaluated in this study. This will need to be further investigated because there is increasing clinical experience with tacrolimus-based immunosuppression in lung transplant recipients. In future experimental and clinical studies, for both tacrolimus- and cyclosporine-based regimens in which sirolimus is added, blood levels of 
these drugs should be measured to assess potential pharmacokinetic interactions.

In summary, lung transplantation is associated with gradually improving survival statistics that have been obtained with both cyclosporine- and tacrolimus-based immunosuppressive regimens. However, obliterative bronchiolitis continues to be the major long-term complication and appears to be a sequela of acute rejection episodes that remains common in lung transplantation. We have evaluated the efficacy of sirolimus as an adjunct to cyclosporine in preventing acute lung rejection in the rat orthotopic lung transplant model. Also, we have shown that the potentiating and cyclosporinesparing effect of sirolimus previously demonstrated in other vascularized organ transplant models is relevant to an experimental lung transplant model. However, we could not demonstrate the efficacy of sirolimus monotherapy as has been suggested by prior studies in nonpulmonary models. In light of the results of this study, combined with increasing clinical experience and safety of sirolimus in human renal transplantation studies, we believe that the combination of sirolimus and cyclosporine may be very promising for clinical lung transplantation.

We thank Suren N. Sehgal, PhD (Wyeth-Ayerst Research Laboratories), for his assistance with providing sirolimus and for his expertise.

\section{REFERENCES}

1. Stepkowski SM, Chen H, Daloze P, Kahan BD. Rapamycin, a potent immunosuppressive drug for vascularized heart, kidney, and small bowel transplantation in the rat. Transplantation 1991; 51:22-6.

2. Morris RE. In vivo immunopharmacology of the macrolides FK506 and rapamycin: toward the era of rational immunosuppressive drug discovery, development, and use. Transplant Proc 1991;23:2722-4.
3. Dumont FJ, Staruch MJ, Koprak SL, et al. Distinct mechanism of suppression of murine $\mathrm{T}$ cell activation by the related macrolides FK506 and rapamycin. J Immunol 1990;144:251-2.

4. Stepkowski SM, Chen H, Daloze P, Kahan BD. Prolongation by rapamycin of heart, kidney, pancreas and small bowel allograft survival in rats. Transplant Proc 1991;23:507-8.

5. Marck KW, Wildevuur CRH. Lung transplantation in the rat: technique and survival. Ann Thorac Surg 1982;34:74-80.

6. Prop J, Ehrie MG, Crapo JD, Nieuwenhuis NCP, Wildevuur CRH. Reimplantation response in isografted rat lungs: analysis of causal factors. J Thorac Cardiovasc Surg 1984;87:702-11.

7. Berry GJ, Brunt EM, Chamberlain D, Hruban RH, Sibley RK, Stewart S, et al. A working formulation for the standardization of nomenclature in the diagnosis of heart and lung rejection: Lung Rejection Study Group. J Heart Lung Transplant 1990;9:593601.

8. Keenan RJ, Konishi H, Kawai A, Paradis IL, Nunley DR, Iacono AT, et al. Clinical trial of tacrolimus versus cyclosporine in lung transplantation. Ann Thorac Surg 1995;60:580-5.

9. Bando K, Paradis IL, Similo S, Konishi K, Konatsu K, Zullo TG, et al. Obliterative bronchiolits after lung and heart-lung transplantation: an analysis of risk factors and management. J Thorac Cardiovasc Surg 1995;110:4-14.

10. Keller CA, Cagle PT, Brown RW, Noon G, Frost A. Bronchiolitis obliterans in recipients of single, double, and heart-lung transplantation. Chest 1995;107:973-80.

11. Ochiai T, Gunji Y, Nagata M, Komori A, Asano T, Isono K. Effects of rapamycin in experimental organ allografting. Transplantation 1993;5615-9.

12. Chen H, Wu J, Luo H, Daloze P. Synergistic effect of rapamycin and cyclosporine in pancreaticoduodenal transplantation in the rat. Tranplant Proc 1992;24:892-3.

13. Kahan BD, Gibbons S, Tejpal N, Strepkowski SM, Chou T-C. Synergistic interactions of cyclosporine and rapamycin to inhibit immune performances of normal human peripheral blood lymphocytes in vitro. Transplantation 1991;51:232-9.

14. Knight R, Ferraresso M, Serino F, Katz S, Lewis R, Kahan BD. Low-dose rapamycin potentiates the effects of subtherapeutic doses of cyclosporine to prolong renal allograft survival in the mongrel canine model. Transplantation 1993;55:947-9.

15. Strepkowski SM, Kahan BD. Rapamycin and cyclosporine synergistically prolong heart and kidney allograft survival. Transplant Proc 1991;23:3262-4. 